\title{
Características Fisicoquímicas y Sensoriales del Néctar de Cocona (Solanum sessiliflorum Dunal) con Extracto de Stevia (Stevia rebaudiana Bertoni).
}

\author{
Physical Chemical and Sensory Characteristics of the Cocona Nectar (Solanum \\ sessiliflorum Dunal) With Stevia Extract (Stevia rebaudiana Bertoni). \\ ${ }^{1}$ Irma Rumela Aguirre Zaquinaula, ${ }^{2}$ Helí Humberto Aguirre Zaquinaula
}

\section{RESUMEN}

Para evaluar el efecto de la concentración de extracto de Stevia rebaudiana Bertoni y sacarosa sobre las características físicoquímicas y sensoriales de néctar de cocona. Se estudió siete tratamientos con tres concentraciones de stevia y cuatro de sacarosa. El tratamiento, con $0.3 \%$ extracto de stevia tuvo $6.47^{\circ}$ Brix de sólidos solubles, el segundo con $0.5 \%$ de extracto de stevia, tuvo $6.58^{\circ}$ Brix de sólidos solubles y el tercer tratamiento con $0.7 \%$ de extracto de stevia, tuvo $6.70^{\circ}$ Brix contenido de sólidos solubles, el cuarto con $0.3 \%$ de concentración de sacarosa tuvo $14^{\circ}$ Brix de sólidos solubles, el quinto tratamiento con $0.5 \%$ de concentración de sacarosa tuvo $16^{\circ}$ Brix de sólidos solubles, el sexto con $0.7 \%$ de concentración de sacarosa tuvo $18^{\circ}$ Brix de sólidos solubles, el séptimo tratamiento con $0.0 \%$ de concentración de sacarosa tuvo $5^{\circ}$ Brix de sólidos solubles. Los resultados se analizaron con la prueba de Friedman determinándose que no existe efecto de la proporción del extracto de stevia y concentración de sacarosa sobre las características sensoriales del néctar de cocona (significancia de $5 \%)$. Se realizó la prueba de Duncan $(\alpha=0.05)$. Los tratamientos con $0.3 \%, 0.5 \%$ y $0.7 \%$ de extracto de stevia y concentración de sacarosa, así como $0.0 \%$ de concentración de sacarosa difieren significativamente en sus valores de $\mathrm{pH}$ y acidez. En cuanto a los grados ${ }^{\circ} \mathrm{Brix}$, los siete tratamientos difieren significativamente. Los mejores tratamientos fueron: $\mathrm{S}_{3}$ con $0.7 \%$ de extracto de stevia y $\mathrm{S}_{6}$ con extracto de sacarosa.

Palabras clave: Néctar, cocona (Solanum sessiliflorum Dunal), stevia (Stevia rebaudiana Bertoni), análisis sensorial.

\begin{abstract}
To evaluate the effect of the concentration of Stevia rebaudiana Bertoni extract and sucrose on the physicochemical and sensorial characteristics of cocona nectar. Seven treatments were studied with three concentrations of stevia and four of sucrose. The treatment, with $0.3 \%$ stevia extract had $6.47^{\circ}$ Brix of soluble solids, the second with $0.5 \%$ stevia extract, had $6.58^{\circ}$ Brix of soluble solids and the third treatment with $0.7 \%$ stevia extract, had $6.70^{\circ}$ Brix content of soluble solids, the fourth with $0.3 \%$ sucrose concentration had $14^{\circ}$ Brix of soluble solids, the fifth treatment with $0.5 \%$ sucrose concentration had $16^{\circ}$ Brix of soluble solids, the sixth with $0.7 \%$ sucrose concentration had $18^{\circ}$ Brix of soluble solids, the seventh treatment with $0.0 \%$ sucrose concentration had $5^{\circ}$ Brix of soluble solids. The results were analyzed with the Friedman test determining that there is no effect of the proportion of the stevia extract and sucrose concentration on the sensory characteristics of the cocona nectar $(5 \%$ significance). The Duncan test was performed $(\alpha=0.05)$. Treatments with $0.3 \%, 0.5 \%$ and $0.7 \%$ stevia extract and sucrose concentration, as well as $0.0 \%$ sucrose concentration differ significantly in their $\mathrm{pH}$ and acidity values. Regarding the degrees ${ }^{\circ}$ Brix, the seven treatments differ significantly. The best treatments were: S3 with $0.7 \%$ stevia extract and S6 with sucrose extract.
\end{abstract}

Keywords: Néctar, cocona (Solanum sessiliflorum Dunal), stevia (Stevia rebaudiana Bertoni), sensory analysis.

\footnotetext{
${ }^{1}$ Universidad Nacional de Jaén. Jaén, Cajamarca. Perú.

${ }^{2}$ Universidad Nacional Toribio Rodríguez de Mendoza. Chachapoyas, Amazonas. Perú.
} 


\section{INTRODUCCIÓN}

La cocona es cultivada principalmente en el Amazonas Occidental, ya sea de Brasil, Colombia y Perú, y se está produciendo cada vez menos en el Ecuador y Venezuela.

En Perú, las regiones de Iquitos y Pucallpa son las mayores productoras y los mayores mercados. El fruto in natura es comercializado en las ferias y mercados de los bajos Ríos Ucayali y Huallaga, como también a lo largo del Río Marañón, y se encuentra fácilmente en las fuentes de soda, restaurantes y hoteles en forma de jugos y helados. Existe una pequeña industria en Pucallpa que produce jugos y néctares para el mercado nacional, principalmente Lima. Según los propietarios de esta industria, ellos no procesan más cantidad de cocona porque no hay oferta de frutos. (Ribeiro, 1990, p. 11-26)

La cocona es un arbusto herbáceo de 1 a $2 \mathrm{~m}$ de altura, erecto, ramificado, que puede vivir hasta tres años en condiciones muy favorables. Las raíces laterales de las plantas pueden extenderse hasta 1,4 m del tronco. (Pahlen, 1977, p. 7:301107).

Las hojas son simples, alternas, con estípulas en forma de espiral, en grupos de tres, largas pecioladas, membranáceas, margen lobadadentada, base asimétrica, y ápice agudo. Las hojas mayores tienen pecíolos de hasta $14 \mathrm{~cm}$ de largo y láminas de hasta $58 \mathrm{~cm}$ de largo. El lado dorsal es de color ceniza, la ventral cubierta por pubescencia es una sustancia aparentemente azucarada que atrae Himenópteros (Apidae, Vespidae, Formicidae) y Dípteros.

La inflorescencia es una cima situada en las ramas entre cada grupo de tres hojas y contiene entre cinco y ocho flores, de las cuales subsisten de uno a tres frutos. La cima está constituida por una rama de poco más de un centímetro, en la cual se ubican, en forma espirada, los pedúnculos florales, cada uno de los cuales mide entre 2 a $5 \mathrm{~mm}$ de largo. La corola es de forma estrellada con 5 pétalos de color verde claro ligeramente amarillento. El cáliz está constituido por 5 sépalos de color verde. Las 5 anteras son amarillas, cada una de $3 \mathrm{~mm}$ de largo y $1 \mathrm{~mm}$ de ancho. (Paytan, 1997, p. 71-76).

Las flores, tanto las hermafroditas como las estaminadas, no poseen diferencias morfológicas externas importantes. Las flores estaminadas poseen estilete reducido y ovario rudimentario. Las flores hermafroditas poseen un estigma húmedo y estilete glabro, midiendo de 7 a $10 \mathrm{~mm}$, y su ovario es piloso y con forma de globo. La floración de la cocona se inicia a los 4 ó 5 meses después de la siembra. Las flores abren alrededor de las 07:00 h y comienzan a cerrar a las 16:00 h. Cuando abren, las anteras están dehiscentes y los estigmas, de un modo general, receptivos.

Las flores duran sólo dos días y si no hay fertilización marchitan y se caen. Al hacer la prueba con hidróxido de amonio, para verificar su grado de reflexión a la luz ultra-violeta, las flores presentan color café intenso en las anteras y pétalos, pero no en los nervios de los pétalos. Exhalan un olor suavemente perceptible al olfato humano. Utilizando rojo neutro, es posible observar que el ápice de las anteras, estigma y bordes de los pétalos colorean suavemente con esta sustancia, evidenciando osmóforos en estas regiones. (Storti, 1988, p.18:5668). La Cocona (Solanum sessiliflorum) es una especie tropical que se originó en las laderas orientales de los Andes del Perú, Colombia, Ecuador y Venezuela y la parte amazónica de Brasil. Se cultiva entre 200 y $1000 \mathrm{~m}$ de altitud. Es una hierba semi perenne que se vuelve semi arbolada con la edad y puede alcanzar los $2 \mathrm{~m}$ de altura. Los frutos redondos, ovales u ovoides son climatérgicos y se consumen crudos o se utilizan para preparar jugos, mermeladas, jaleas, salsas calientes y encurtidos, y también se utilizan en la medicina popular. Los frutos son duros y pueden soportar un manejo bastante brusco después de la cosecha. Comercialmente es mejor como un cultivo anual con el fin de maximizar los rendimientos. (Science, Technology and Nutrition. 2011, p. 1-7, 80). 
El fruto es una entidad estructural procedente de la transformación de la flor, como consecuencia del desarrollo de los tejidos que soportan los óvulos de la planta. Después de la polinización de la flor los primordios seminales dan lugar a las semillas y todo el ovario sufre una serie de reproducciones celulares, volviéndose criboso, endurecido cambiando de forma. El carpelo se diferencia en epicarpio, mesocarpio, endocarpio, que corresponden a la parte externa, media e interna, respectivamente. La cocona (Solanum $s p$.) es una planta herbácea de la Amazonia cuyo centro de origen se ubica en el alto Orinoco. El fruto varía en su forma de acuerdo al genotipo, y su coloración va de verde (inmaduro) a amarillo o marrón opaco (maduro). (Pronatta, 2001).

El fruto de la cocona es una baya que puede variar desde casi esférica hasta ovalada con 4-12 $\mathrm{cm}$ de ancho y 3-6 cm de largo, peso entre $24 \mathrm{y}$ 250 gramos, color desde amarillo hasta rojizo, cubierta de pubescencia fina y suelta. La pulpa es de sabor ácido, aroma similar al del tomate de árbol y color claro amarillo cremoso; generalmente representa entre el 75 y $82 \%$ del peso total del fruto. Los indicadores de madurez del fruto de cocona más importantes son los parámetros de crecimiento longitud y diámetro, los cambios de color del fruto, que cambia a naranja casi en la totalidad de su superficie, la firmeza del fruto que oscila entre 9-10 libras y la relación de madurez ( ${ }^{\circ} \mathrm{Brix} / \%$ acidez) de 11 . El volumen del jugo es de hasta $36 \mathrm{~cm} 3 /$ fruto y el grado Brix de 4-6. (Pronatta, 2001).

Para desarrollar un buen proceso de industrialización o transformación, se debe escoger el material que presente las mejores características específicas para el objetivo que se ha propuesto en el procesamiento. Los constituyentes de una fruta dulce se dividen en dos grandes grupos: los minerales y los orgánicos. En cuanto a los minerales son de importancia el agua como elemento dispersante, electrolítico, y las sales minerales fundamentales como cofactores enzimáticos en los procesos bioquímicos metabólicos, tanto para la fruta (maduración) como para el resto del círculo trófico derivado (alimentación y nutrición). Los orgánicos están conformados principalmente por hidratos de carbono, lípidos, proteínas, aminoácidos, ácidos orgánicos, pigmentos, aromas, vitaminas y hormonas. En términos generales los frutos de cocona son frutos catalogados como ácidos, ricos en agua y minerales como potasio y calcio, y constituyen una fuente energética de importancia debido al alto contenido de carbohidratos. $\mathrm{Su}$ aporte en grasa es medio, al igual que en vitamina $\mathrm{C}$ y en hierro. (Pronatta, 2001).

La stevia en particular es un aditivo alimentario bajo en calorías o podría llamarse así el fármaco potencial adecuado para los diabéticos (YongHeng et al., 2014).

Los alimentos procesados contienen glucosidos de esteviol que son bajos en calorías, además su dulzor es de 100 a 300 veces mayor que el de la sacarosa (Lemus Mondaca et al., 2012), mientras que el del rebaudiósido A es unas 50 a 250 veces superior. Estos glucósidos no pueden ser absorbidos en el tracto gastrointestinal, por lo que son hidrolizados principalmente por bacilos del grupo Bacteroides de la microbiota intestinal (Renwick y Tarka, 2008).

Mantiene los niveles de glucosa porque no se ingiere calorías, regula la insulina en la sangre, el organismo engorda menos. Las hojas de la planta silvestre de Estevia contienen 0.3\% Dulcósido, $0.6 \%$ Rebaudiósido C, 3.8\% Rebaudiósido A y el 9.1\% de Esteviósido. (ICTA, 1986, p. 325).

Sin embargo, al sustituir los edulcorantes en un producto pueden cambiar los sabores, olores y/o textura del producto debido al diferencial de 
sólidos por la proporción de edulcorante en los productos, es por esto que es necesario saber la combinación o uso necesario de edulcorantes para minimizar el cambio en sabor del producto obteniendo los resultados de reducción calórica que se buscan (Bello, 2000).

Estudios señalan que el extracto de hojas de stevia actúa como bactericida sobre Streptococcus mutans, responsable de las caries dentales al poseer propiedades antibacterianas y antivirales. (Kujur, 208, p.258-263).

Tabla 1. Composición química de la cocona (Solanum sessiliflorum) en $100 \mathrm{~g}$ de pulpa integral (Pahlen, 1977; Andrade et al., 1996; Villachica, 1996; Yuyama et al., 1997, 1998).

\begin{tabular}{|l|l|l|l|l|}
\hline Componente & Villachica & Pahlen & Andrade & Yuyama \\
\hline & & & & \\
\hline Humedad (g) & 89 & 91 & 93 & 90 \\
\hline Energía(g) & 41 & 33 & 31 & 45 \\
\hline Proteina(g) & 0,9 & 06 & - & 0,9 \\
\hline Lípidos (g) & - & 1,4 & - & - \\
\hline Extacto libre de N (g) & - & 5,7 & - & - \\
\hline Fibra (g) & 0,2 & 0,4 & - & 1,6 \\
\hline Cenizas (g) & 0,7 & 0,9 & - & 0,9 \\
\hline Azucares totales (\%) & - & - & 4,6 & - \\
\hline Azucares reductores (\%) & - & - & 3,9 & - \\
\hline Azucares no reductores (\%) & - & - & 1,8 & - \\
\hline Sólidos solubles (\%) & - & 5,0 & 8,0 & - \\
\hline Ácido Cítrico (\%) & - & - & 0,8 & - \\
\hline Brix/ Ácidez & - & - & 5,93 & - \\
\hline Compuestos fenolicos (mg) & - & - & 14,4 & - \\
\hline Taninos (mg) & - & - & 142 & - \\
\hline
\end{tabular}

\section{MATERIALY MÉTODOS}

\section{Materia prima}

La pulpa es de sabor ácido, aroma similar al del tomate de árbol y color claro amarillo cremoso; generalmente representa entre el 75 y $82 \%$ del peso total del fruto. El néctar deberá ser extraído de coconas maduras, sanas y frescas, convenientemente lavadas y libres de restos de plaguicidas y otras sustancias nocivas, en condiciones sanitarias apropiadas. Una de las ventajas de la elaboración de este producto es que la forma de procesamiento permite el empleo de frutas que no son adecuadas para otros fines por su forma y tamaño.

La cocona tiene un sabor muy característico que no se puede comparar con el sabor de otras frutas. No obstante, algunas personas dicen que se parece al sabor del tomate y limón juntos, lo que tiene sentido ya que la cocona perdió su importancia cuando se introdujo estas dos especies en el Amazonas. Aunque sea de la familia de la naranjilla, su sabor es distinto.

Sus nombres en inglés, Orinoco apple y peach tomato, tampoco se refieren al sabor, pues no se parece al sabor de la manzana y del durazno. La mayor semejanza está en la forma y en el color de la pulpa.

La pulpa de la placenta es ligeramente más ácida y mucho más sabrosa que la pulpa adherida a la cáscara. En algunas etnovariedades, la pulpa presenta un sabor suavemente amargo, que puede ser en función del suelo o del agua con la cual se riega. Debido a la baja relación sólidos solubles/acidez. (Andrade, 1977, p. 115).

La cocona presenta poco grado de azúcar. Por esto, el fruto es raramente consumido in natura, excepto como complemento de bebidas alcohólicas.

La preparación de jugos, dulces, mermeladas y compotas es el principal uso de los frutos. Los frutos también pueden ser consumidos en forma de salsa para acompañar asado de corazón de vacuno (conocido en el Amazonas peruano como "anticucho") y en las sopas de pescado (popularmente denominadas de "caldeirada" o "peixada" en el Amazonas brasileño). 


\section{Procedimiento}

\section{A. Recepción}

Se utilizó como materia prima la cocona, (Solanum sessiliflorum Dunal) traídas del distrito de San José de Lourdes, de la provincia de San Ignacio. Los frutos se manipularon con cuidado, evitando golpearlos, los cuales entraron al laboratorio de industrias alimentarias y a ser procesados. Se pesó la materia prima, con el uso de una balanza semianalítica para posteriormente hacer el cálculo de rendimiento.

\section{B. Selección y Clasificación}

Se seleccionaron las cocona, (Solanum sessiliflorum Dunal) en buen estado físico, es decir, sin magulladuras ni golpes; además se tomó en cuenta la madurez sensorial del fruto en cuanto al olor y color.

\section{Lavado y Desinfección:}

La desinfección tiene como fin disminuir al máximo la contaminación de microorganismos que naturalmente trae la cáscara de la fruta. Para la desinfección se utiliza hipoclorito de sodio en solución, con una concentración de 50 ppm de cloro. En las etapas de lavado y desinfección de la fruta es importante destacar la importancia del manejo óptimo del agua con el fin de minimizar los costos de operación por servicios; para tal fin es importante recircular el agua resultante en las etapas de desinfección (solución desinfectante) y aspersión, ya que el principal propósito de la etapa de prelavado es retirar la tierra y otros elementos indeseables que disminuyen la calidad de la fruta. El lavado de la materia prima se llevó a cabo en forma manual bajo fricción y con agua potable con la finalidad de eliminar suciedad, pelusa $\mathrm{y} / \mathrm{o}$ restos de tierra adheridos en la superficie de la fruta.

\section{Acondicionamiento}

La cocona se cortó en pequeños trozos con cuchillos de acero inoxidable sobre tablas de picar en forma manual extrayendo las semillas. Inmediatamente que son cortados ingresan a la etapa siguiente para evitar que la materia prima pueda pardearse.

\section{E.Precocción}

Los trozos de cocona fueron colocados en agua a $100^{\circ} \mathrm{C}$ por 5 minutos para ablandarlos e inactivar las enzimas que oscurecen y que incluso modifican el sabor.

\section{F. Pulpeado}

Se define como pulpa de frutas el producto pastoso, no diluido, ni concentrado, ni fermentado obtenido por la desintegración y tamizado de la fracción comestible de frutas frescas, sanas, maduras y limpias. (Pronatta, 2001). Las características y comportamientos de las pulpas de frutas están íntimamente relacionadas con la especie, variedad, grado de madurez y las condiciones ambientales durante el desarrollo de la fruta. Los trozos de cocona se enfriaron al ambiente por 20 minutos para luego licuarlos. (Camacho, 1998).

\section{G. Homogenización}

Este proceso consistió en dar un tratamiento mecánico a las partículas obtenidas del pulpeado con el fin de obtener una pulpa más suave y uniforme utilizando para ello un molino coloidal.

\section{H. Estandarización}

Una vez obtenida la pulpa pura de cocona 1:3 pulpa: agua, se endulzó con extracto de Stevia (edulcorante natural) en tres proporciones diferentes de $0.3 \%, 0.5 \%$ y $0.7 \%$, y extracto de sacarosa en cuatro proporciones diferentes de 0.3 $\%, 0.5 \%$ y $0.7 \%, 0.0 \%$, se añadió $0.07 \%$ de Carboximetilcelulosa (CMC) y $0.05 \%$ de Sorbato de Potasio (Figura 9). No fue necesario adicionar ácido cítrico, puesto que la mezcla llegó a un pH de 3.5, el cual se encontró en un rango óptimo para el proceso posterior de pasteurización. (Pronatta, 2001). 


\section{Pasteurización}

Se consideró siete tratamientos, sometidos a una temperatura de $85{ }^{\circ} \mathrm{C}$ por 15 minutos. Con la finalidad de reducir la carga microbiana $\mathrm{y}$ asegurar la inactividad del néctar.

\section{J. Envasado}

El llenado se realizó en botellas de vidrio de capacidad de $250 \mathrm{ml}$ con ayuda de un embudo evitando la formación de espuma a una temperatura no menor a $80^{\circ} \mathrm{C}$ y luego se colocó la tapa en forma manual.

\section{K. Enfriamiento}

Las botellas se dejaron enfriar a temperatura ambiente y luego con agua corriente hasta temperatura ambiente. Los siete tratamientos de néctar de cocona fueron almacenados a $3^{\circ} \mathrm{C}$ por 5 días para ser sometidos a evaluación sensorial y fisicoquímica.

\section{L.Almacenamiento}

Los siete tratamientos de néctar de cocona endulzados con extracto de stevia y extracto de sacarosa fueron almacenados a temperatura de refrigeración $\left(5^{\circ} \mathrm{C}\right)$ por cinco días y asegurar el mantenimiento de sus características hasta la realización del análisis fisicoquímico y sensorial.

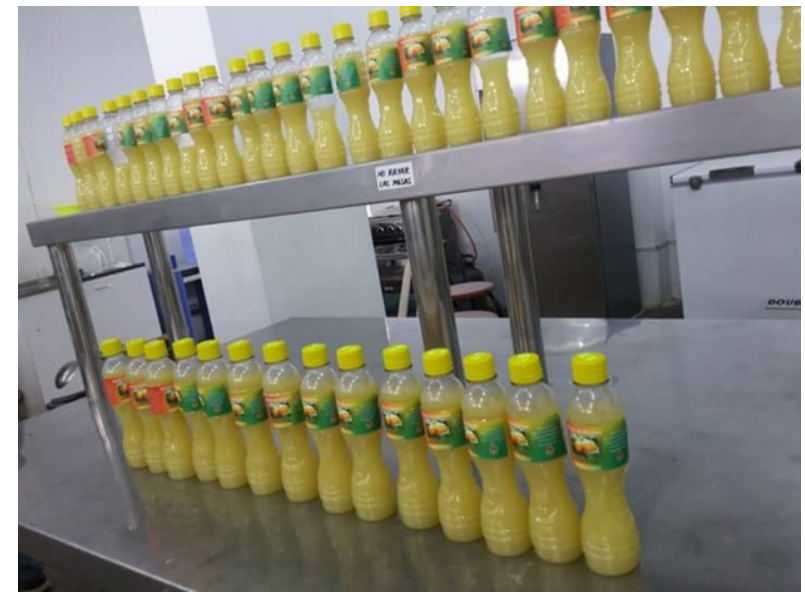

Figura 1. Almacenamiento del néctar a temperatura ambiente.

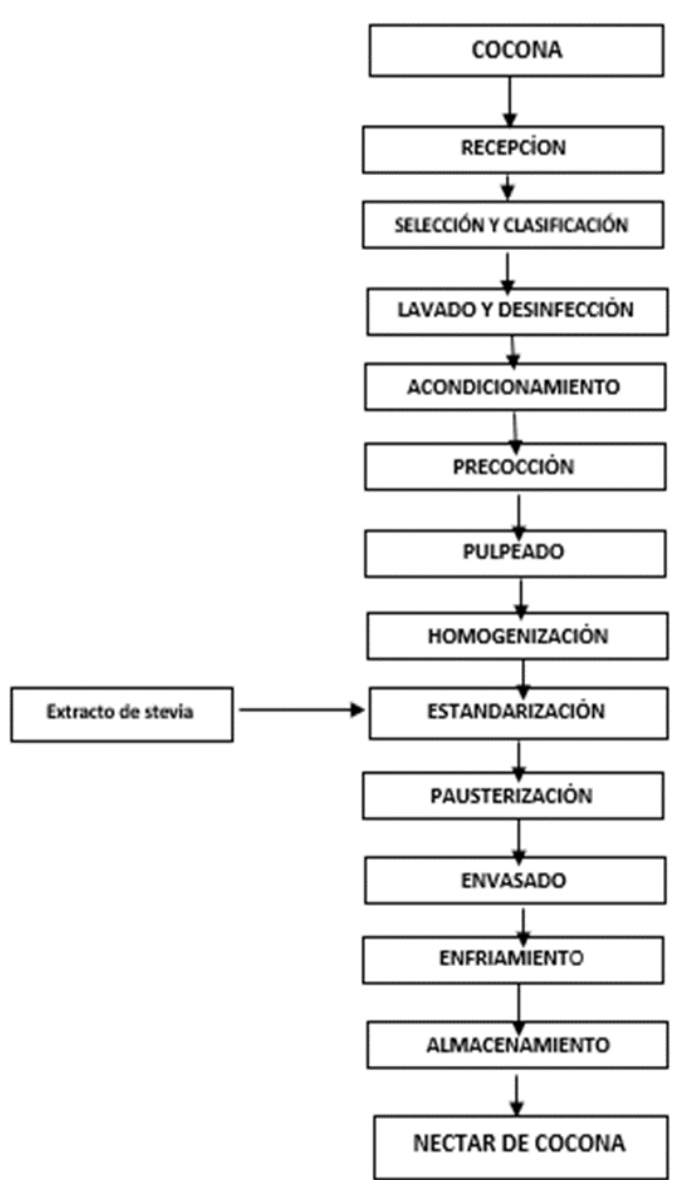

Figura 2. Flujo experimental de la Elaboración de néctar de cocona (Solanum sessiliflorum Dunal) endulzado con extracto de stevia (Stevia Rebaudiana Bertoni) y concentración de sacarosa.

\section{Análisis fisicoquímico del néctar de cocona}

Se realizó el análisis físico-químico en el néctar de cocona para determinar: brix, $\mathrm{pH}, \mathrm{y}$ acidez títulable.

\section{Determinación de sólidos solubles}

Refractometría(INTINTEC, 1989)

\section{Determinación de pH}

Método: Potenciométrico (EGAN, 1995)

Evaluación de las diferencias de potencial entre un electrodo estándar de calomel previamente calibrados usando sales amortiguadoras.

\section{Determinación de acidez total}

Método: acidez títulable (A.O.A.C, 1995)

Neutralización de la acidez producida por la 
muestra en dilución acuosa con soda utilizando fenolftaleína como indicador.

\section{Análisis Estadístico}

Se evaluó las características fisicoquímicas de los siete tratamientos de néctar de cocona endulzado con extracto de stevia y concentración de sacarosa, utilizando un diseño completamente al azar y una prueba de comparación de mesclas la Duncan para identificar los tratamientos que presentaran diferencias significativas.

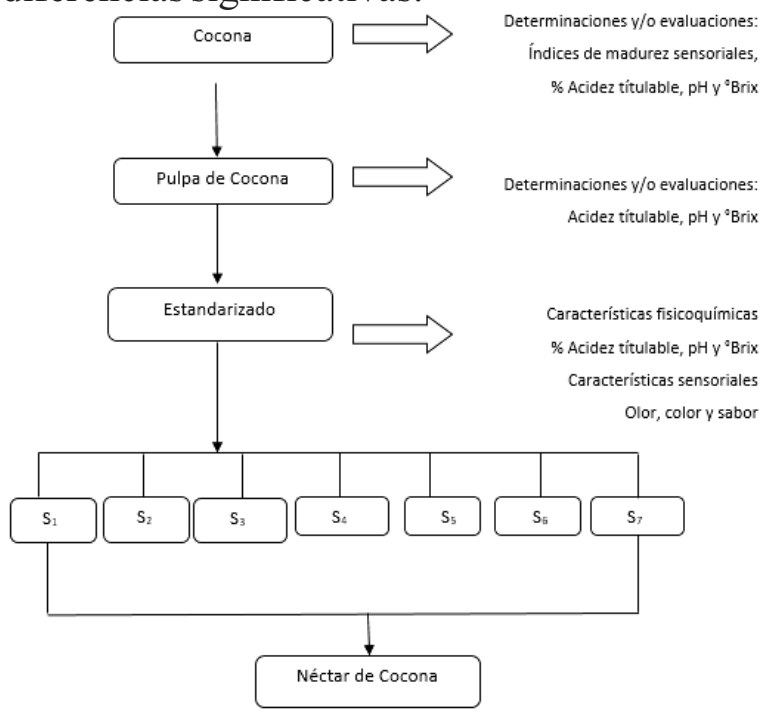

Figura 3. Diseño del experimento para evaluar el efecto de extracto de stevia y concentración de sacarosa en las características fisicoquímicas y sensoriales de néctar de cocona (Stevia rebaudiana Bertoni). Y relación de tratamientos de los experimentos (SI al S7).

\section{LEYENDA}

$\mathrm{S}_{1}: 0.3 \%$ extracto de stevia.

$\mathrm{S}_{2}: 0.5 \%$ extracto de stevia.

$\mathrm{S}_{3}: 0.7 \%$ extracto de stevia.

$\mathrm{S}_{4}: 0.3 \%$ concentración de sacarosa.

$\mathrm{S}_{5}: 0.5 \%$ concentración de sacarosa

$\mathrm{S}_{6}: 0.7 \%$ concentración de sacarosa.

$\mathrm{S}_{7}: 0.0 \%$ concentración de sacarosa.

\section{RESULTADOS Y DIVISIÓN}

\section{Evaluación Sensorial}

A continuación se muestran los resultados de la evaluación sensorial, en la que participaron 30 panelistas de la carrera de Ingeniería Forestal, seleccionados al azar, los cuales calificaron los atributos olor, color y sabor a los siete tratamientos de néctar de cocona endulzados con extracto acuoso de stevia y concentración de sacarosa. El primer tratamiento, con $0.3 \%$ de extracto tuvo un contenido de sólidos solubles equivalente a $6.47^{\circ} \mathrm{Brix}$, el segundo tratamiento con $0.5 \%$ de extracto, un contenido de sólidos solubles equivalente a $6.58{ }^{\circ}$ Brix y el tercer tratamiento con $0.7 \%$ de extracto, un contenido de sólidos solubles equivalente a $6.70^{\circ} \mathrm{Brix}$, el cuarto tratamiento con $0.3 \%$ de concentración de sacarosa tuvo un contenido de sólidos solubles equivalente a $14{ }^{\circ} \mathrm{Brix}$, el quinto tratamiento con $0.5 \%$ de concentración de sacarosa tuvo un contenido de sólidos solubles equivalente a 16 ${ }^{\circ}$ Brix , el sexto tratamiento con $0.7 \%$ de concentración de sacarosa tuvo un contenido de sólidos solubles equivalente a $18{ }^{\circ} \mathrm{Brix}$, el séptimo tratamiento con $0.0 \%$ de concentración de sacarosa tuvo un contenido de sólidos solubles equivalente a $5{ }^{\circ}$ Brix. En la tabla 1 se puede observar que el mayor puntaje total otorgado por los panelistas en cuanto al atributo color de extracto de stevia fue para el tratamiento $\mathrm{S}_{3}(0.7 \%$ de extracto de stevia) con 204 puntos, seguido del tratamiento $S_{2}(0.5 \%$ de extracto de stevia) con 168 puntos y finalmente con una muy poca diferencia el tratamiento $\mathrm{S}_{1}$ ( $0.3 \%$ de extracto de stevia) con 156 puntos. El mayor puntaje total otorgado por los panelistas en cuanto al atributo color de extracto de sacarosa fue para para el tratamiento $\mathrm{S}_{6}(0.7 \%$ de extracto de sacarosa) seguido del tratamiento $\mathrm{S}_{2}(0.5 \%$ de extracto de sacarosa) con 190 puntos y finalmente con una muy poca diferencia el tratamiento $\mathrm{S}_{1}(0.3 \%$ de extracto de sacarosa) con 159 puntos y con diferencia significativa del tratamiento $\mathrm{S}_{7}(0.0 \%$ de extracto de sacarosa) con 66 puntos. 
Tabla 1. Resultados de la prueba de aceptación para el atributo color de los siete tratamientos de néctar de cocona endulzados con extracto de stevia y concentración de sacarosa

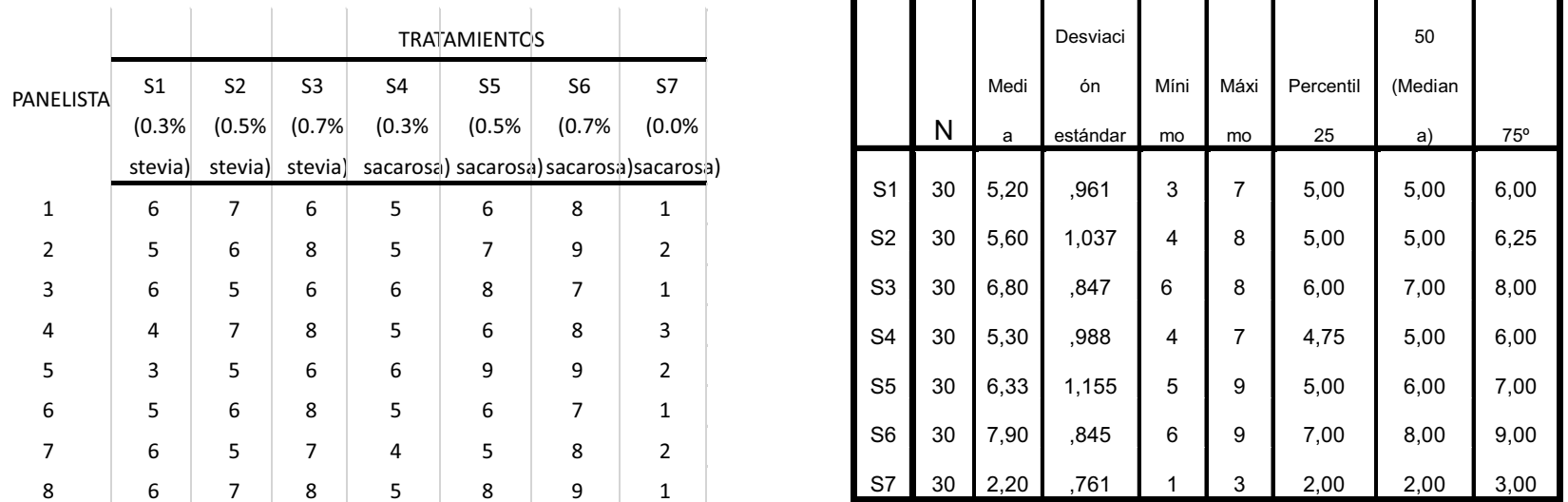

Prueba de Friedman.

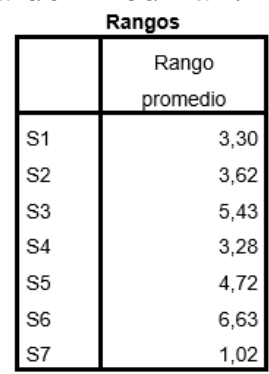

Estadísticos de pruebaal

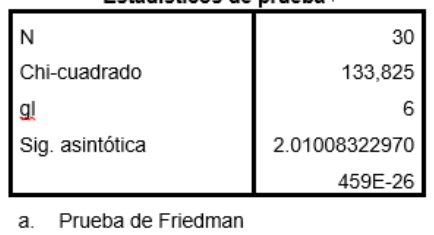

Según la tabla 2 los resultados indican que no hay diferencia significativa entre los tratamientos después de aplicar el test de friedman a un nivel de significancia de 5\% a los resultados obtenidos del análisis sensorial del atributo color $(\mathrm{p}=2.01008322970459 \mathrm{E}$ $26>0.05$ ). Por lo tanto, no hay efecto de la proporción de stevia sobre la característica sensorial de color. A continuación en la tabla 3, se muestran los resultados de la prueba de aceptación realizada para el atributo sensorial de olor a los siete tratamientos de néctar de cocona endulzado con extracto de stevia y concentración de sacarosa. 
Tabla 3. Resultados de la prueba de aceptación para el atributo olor de los siete tratamientos de néctar de cocona endulzados con extracto de steviay concentración de sacarosa.

\begin{tabular}{|c|c|c|c|c|c|c|c|}
\hline \multirow[b]{2}{*}{ PANELISTA } & \multicolumn{7}{|c|}{ TRATAMIENTOS } \\
\hline & $\begin{array}{c}\mathrm{S} 1 \\
(0.3 \% \\
\text { stevia) }\end{array}$ & $\begin{array}{c}\mathrm{S} 2 \\
(0.5 \% \\
\text { stevia) }\end{array}$ & $\begin{array}{c}\text { S3 } \\
\text { (0.7\% stevia }\end{array}$ & $\begin{array}{c}54 \\
\text { ia) } \\
\text { sacarosa) }\end{array}$ & $\begin{array}{c}\text { S5 } \\
(0.5 \% \\
\text { sacarosa) }\end{array}$ & $\begin{array}{c}\mathrm{S6} \\
(0.7 \% \text { sacarosa }\end{array}$ & $\begin{array}{c}\mathrm{S7} \\
\text { a) }(0.0 \% \\
\text { sacarosa) }\end{array}$ \\
\hline 1 & 5 & 6 & 5 & 5 & 5 & 8 & 4 \\
\hline 2 & 6 & 5 & 6 & 5 & 6 & 7 & 5 \\
\hline 3 & 4 & 7 & 7 & 6 & 5 & 8 & 4 \\
\hline 4 & 5 & 5 & 5 & 5 & 6 & 7 & 5 \\
\hline 5 & 6 & 6 & 6 & 6 & 5 & 5 & 4 \\
\hline 6 & 5 & 5 & 7 & 5 & 6 & 7 & 5 \\
\hline 7 & 4 & 6 & 5 & 4 & 4 & 8 & 5 \\
\hline 8 & 5 & 5 & 7 & 5 & 5 & 7 & 4 \\
\hline 9 & 6 & 6 & 5 & 6 & 5 & 8 & 5 \\
\hline 10 & 5 & 5 & 6 & 5 & 4 & 7 & 3 \\
\hline 11 & 4 & 6 & 5 & 4 & 5 & 7 & 5 \\
\hline 12 & 5 & 7 & 7 & 6 & 4 & 8 & 4 \\
\hline 13 & 6 & 6 & 5 & 5 & 6 & 7 & 5 \\
\hline 14 & 5 & 7 & 7 & 4 & 5 & 8 & 3 \\
\hline 15 & 4 & 6 & 5 & 7 & 5 & 7 & 4 \\
\hline 16 & 5 & 7 & 7 & 5 & 7 & 8 & 5 \\
\hline 17 & 6 & 5 & 5 & 6 & 5 & 7 & 3 \\
\hline 18 & 5 & 7 & 7 & 5 & 7 & 8 & 4 \\
\hline 19 & 4 & 5 & 5 & 4 & 5 & 7 & 5 \\
\hline 20 & 5 & 7 & 6 & 6 & 7 & 8 & 4 \\
\hline 21 & 5 & 5 & 7 & 7 & 6 & 7 & 2 \\
\hline 22 & 6 & 7 & 6 & 6 & 6 & 8 & 4 \\
\hline 23 & 5 & 5 & 5 & 7 & 6 & 7 & 3 \\
\hline 24 & 6 & 7 & 8 & 5 & 7 & 8 & 2 \\
\hline 25 & 5 & 5 & 7 & 4 & 7 & 6 & 3 \\
\hline 26 & 6 & 7 & 8 & 4 & 6 & 7 & 2 \\
\hline 27 & 5 & 5 & 5 & 5 & 4 & 8 & 4 \\
\hline 28 & 6 & 7 & 7 & 4 & 5 & 7 & 2 \\
\hline 29 & 5 & 7 & 8 & 6 & 4 & 8 & 4 \\
\hline 30 & 4 & 5 & 6 & 5 & 5 & 8 & 2 \\
\hline promedio & 5 & 6 & 6 & 5 & 5 & 7 & 4 \\
\hline TOTAL & 153 & 179 & 185 & 157 & 163 & 221 & 114 \\
\hline
\end{tabular}

En la tabla 3 se puede observar que el tratamiento $\mathrm{S}_{3}(0.7 \%$ de extracto de stevia) obtuvo el mayor puntaje otorgado por los panelistas con 185 puntos; $\mathrm{y} \mathrm{S}_{7}(0.7 \%$ de extracto de sacarosa) obtuvo el mayor puntaje con 221 puntos. Por lo que el análisis estadístico no paramétrico de test de Friedman fue fundamental para poder determinar la existencia o no de diferencia significativa en cuanto al atributo olor.

A continuación, en la tabla 4 se muestran los resultados del test Friedman aplicado a los resultados del análisis sensorial del atributo olor de los tres tratamientos de néctar de cocona endulzado con stevia.
Tabla 4. Resultado de la prueba de Friedman aplicada a los siete tratamientos para el atributo sensorial de olor.

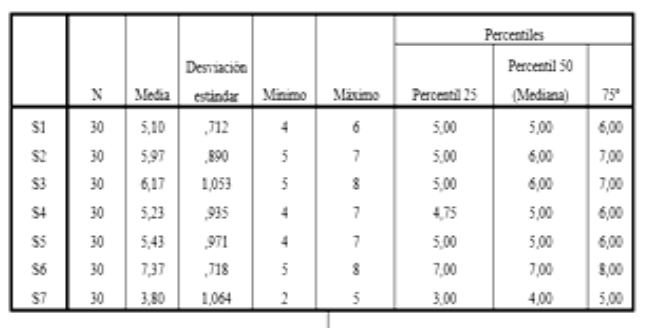

Prueba de Friedman
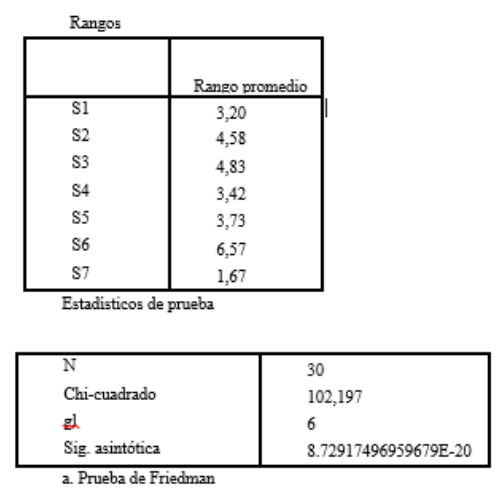

Según la tabla 4 los resultados del análisis estadístico utilizando el test de Friedman a un nivel de significancia de 5\% indican que no hay diferencia significativa entre los tratamientos de néctar de cocona para el atributo sensorial de olor $(p=8.72917496959679 E-20>0.05)$. Por lo tanto la proporción de extracto de stevia no afecta significativamente la característica sensorial de olor. La tabla 5, se muestran los resultados de la prueba de aceptación realizada para el atributo sensorial de sabor a los siete tratamientos de néctar de cocona endulzado con extracto de stevia y sacarosa.

Tabla 5. Resultados de la prueba de aceptación para el atributo sabor de los siete tratamientos de néctar de cocona endulzados con extracto de stevia y sacarosa. 


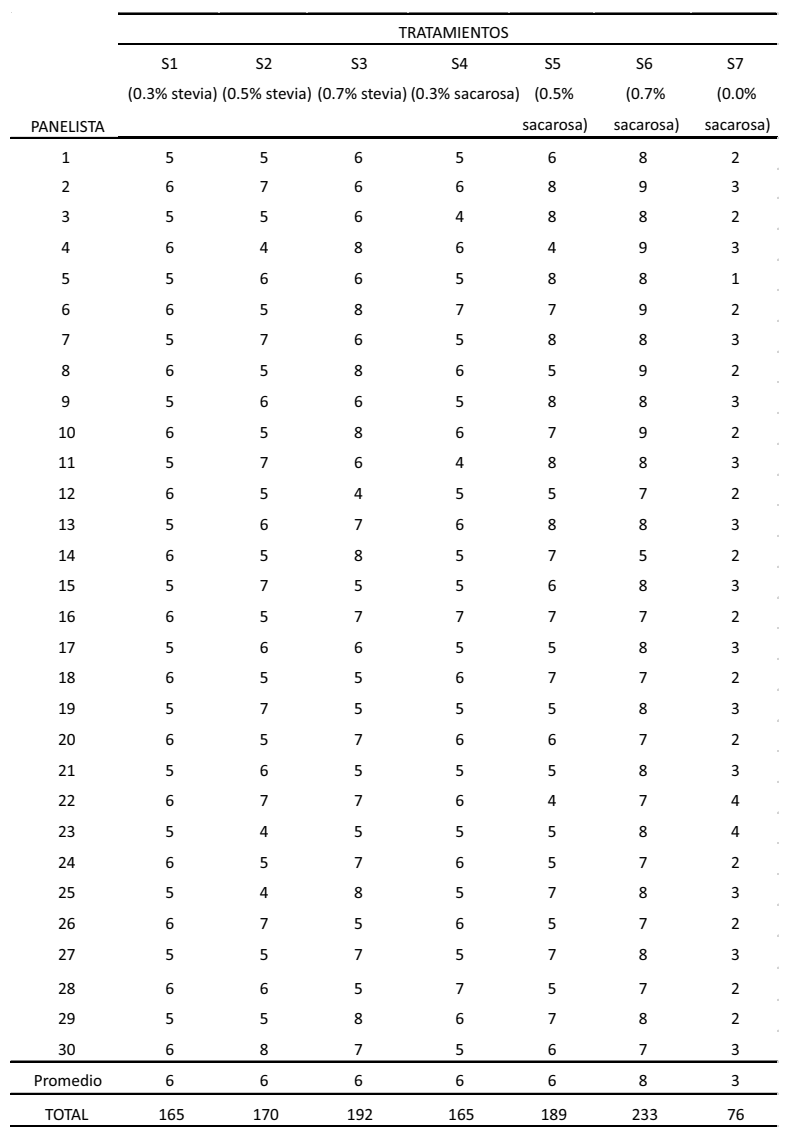

En la tabla 5 se observa que el tratamiento $\mathrm{S}_{3}(0.7$ $\%$ de extracto de stevia) obtuvo el mayor puntaje otorgado por los panelistas con 192 puntos; y $\mathrm{S}_{7}$ ( $0.7 \%$ de extracto de sacarosa) obtuvo el mayor puntaje con 233 puntos. Como se puede observar los resultados no difieren mucho entre los tres tratamientos, por lo que seguidamente se realizó el análisis estadístico a través del test de Friedman.
Tabla 6. Resultado de la prueba de Friedman aplicada a los siete tratamientos.
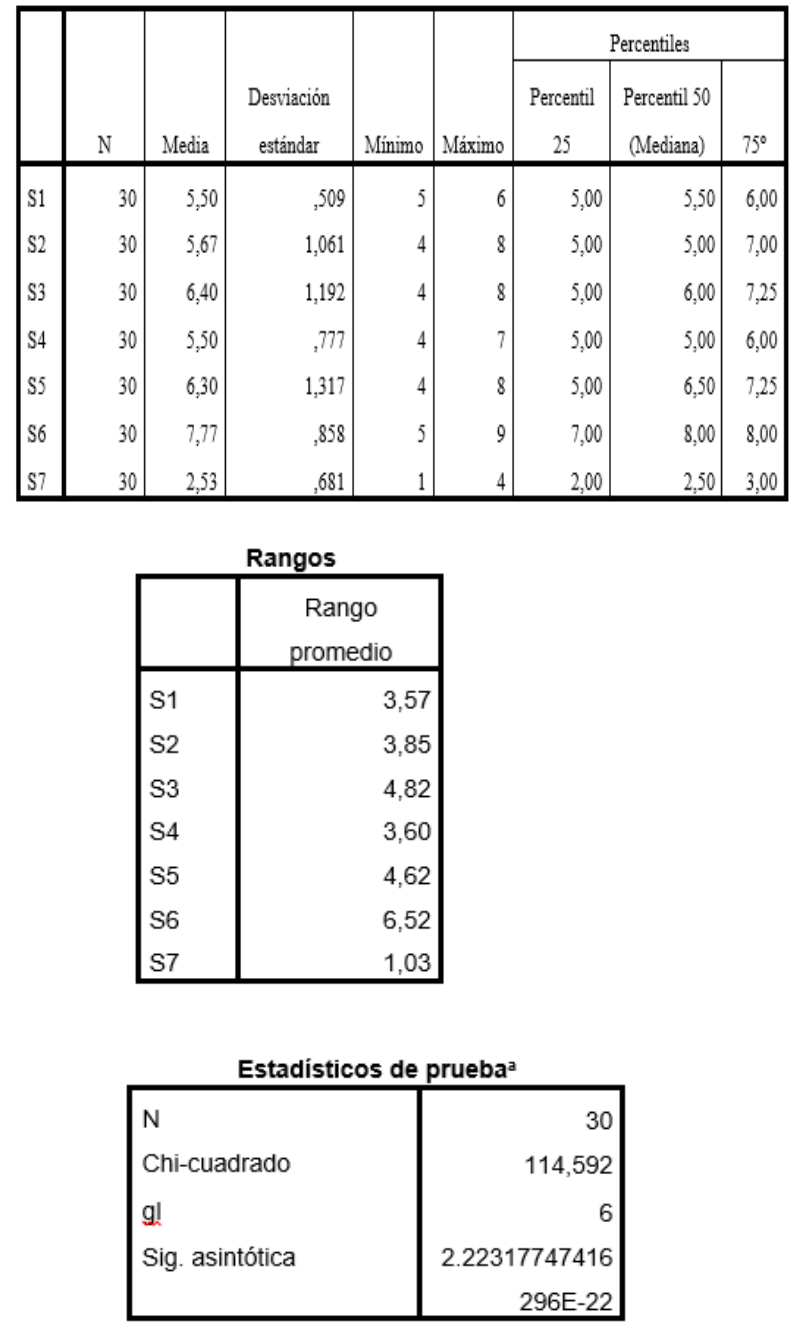

a. Prueba de Friedman

Según la tabla 6 los resultados del análisis estadístico utilizando el test de friedman a un nivel de significancia de 5\% indican que no hay diferencias significativas entre los tratamientos de néctar de cocona para el atributo sensorial de olor $(p=2.22317747416296 \mathrm{E}-22>0.05)$. Por lo tanto la proporción de extracto de stevia no afecta significativamente sobre la característica sensorial de sabor.

A partir de los resultados del test de friedman para los puntajes de color, olor y sabor de los siete tratamientos, como se muestran en las tablas 1,2 y 3 , se concluye que la proporción de extracto de stevia y sacarosa no afecta significativamente las características sensoriales de néctar de cocona. 
Tabla 7. Resultados promedios de cada atributo para el atributo sensorial de sabor.

\begin{tabular}{llllllll}
\cline { 2 - 7 } & \multicolumn{1}{c}{ TRATAMIENTOS } \\
\hline & $\mathbf{S}_{\mathbf{1}}$ & $\mathbf{S}_{\mathbf{2}}$ & $\mathbf{S}_{\mathbf{3}}$ & $\mathbf{S}_{\mathbf{4}}$ & $\mathbf{S}_{\mathbf{5}}$ & $\mathbf{S}_{\mathbf{6}}$ & $\mathbf{S}_{\mathbf{7}}$ \\
\hline color & 5 & 6 & 7 & 5 & 6 & 8 & 2 \\
olor & 5 & 6 & 6 & 5 & 5 & 7 & 4 \\
sabor & 6 & 6 & 6 & 6 & 6 & 8 & 3 \\
\hline
\end{tabular}

Según la tabla 7 , en cuanto al color de los siete tratamientos obtuvieron los siguientes puntajes: $\mathrm{S}_{1} \mathrm{y} \mathrm{S}_{4}$ obtuvieron un puntaje promedio de 5 que significa "no me gusta ni me disgusta ", $\mathrm{S}_{2 y} \mathrm{~S}_{5}$ obtuvieron un puntaje promedio de 6 que significa "me gusta levemente", $\mathrm{S}_{3}$ obtuvo un puntaje promedio de 7 que significa "me gusta moderadamente " $\mathrm{S}_{6}$ obtuvieron un puntaje promedio de 8 que significa "me gusta mucho ", $\mathrm{S}_{7}$ obtuvieron un puntaje promedio de 2 que significa "me disgusta mucho".

En cuanto al olor de los siete tratamientos obtuvieron los siguientes puntajes: $\mathrm{S}_{1}, \mathrm{~S}_{4}, \mathrm{~S}_{5}$ obtuvieron un puntaje promedio de 5 que significa "no me gusta ni me disgusta ", $\mathrm{S}_{2}$ y $\mathrm{S}_{3}$ obtuvieron un puntaje promedio de 6 que significa "me gusta levemente", s6 obtuvieron un puntaje promedio de 7 que significa "me gusta moderadamente ", $\mathrm{S}_{7}$ obtuvieron un puntaje promedio de 3 que significa "me disgusta moderadamente".

En cuanto al sabor los tratamientos $\mathrm{S}_{1}, \mathrm{~S}_{2}, \mathrm{~S}_{3}, \mathrm{~S}_{4}$, $\mathrm{S}_{5}$ obtuvieron un puntaje promedio de 6 que significa "me gusta levemente"; sin embargo el tratamiento $\mathrm{S}_{7}$ obtuvo un puntaje de 3 , es decir "me disgusta moderadamente"; sin embargo como se mencionó antes, no hay diferencia significativa entre los tratamientos para las características sensoriales de color, olor y sabor.

\section{Caracterización fisicoquímica}

\subsection{Caracterización del fruto fresco de cocona}

La materia prima proveniente del distrito de San José de Lourdes, Provincia de San Ignacio, Departamento de Cajamarca ingresó al laboratorio de industrias alimentarias para ser procesada. A continuación en la Tabla 8 se muestran características de la materia prima utilizada en la elaboración del néctar.

Tabla 8. Índices de madurez sensoriales

\begin{tabular}{ll}
\hline $\begin{array}{l}\text { Características } \\
\text { físicas }\end{array}$ & Descripción \\
\hline Color de piel & Anaranjado intenso \\
Color de pulpa & Amarillo claro \\
Sabor & ácido \\
Textura & Firme \\
Olor & Aromático \\
& $4 \mathrm{~cm}-12 \mathrm{~cm}$ de ancho x 3cm- \\
Tamaño & $6 \mathrm{~cm}$ de largo \\
Forma Periforme & Esférica hasta ovalada \\
\hline
\end{tabular}

A continuación, en la Tabla 9 se muestran los resultados obtenidos del análisis físicoquímico de $\mathrm{pH}$, acidez titulable (\% de ácido cítrico) y ${ }^{\circ}$ Brix del fruto fresco de cocona utilizado como materia prima para la elaboración del néctar de cocona.

Tabla 9. Índices de madurez sensoriales

\begin{tabular}{|c|c|}
\hline Características físicoquímicas & Resultado \\
\hline${ }^{\circ}$ Brix & 5 \\
\hline $\begin{array}{l}\text { Acidez titulable ( } \% \text { de ácido } \\
\text { cítrico) }\end{array}$ & 1.6 \\
\hline
\end{tabular}

Fuente: Elaboración propia.

A continuación en la Tabla 10, se muestran los resultados del análisis fisicoquímico de $\mathrm{pH}, \%$ de acidez titulable ${ }^{\circ}$ Brix del jugo de cocona.

Tabla 10. Características fisicoquímicas del jugo de cocona

\begin{tabular}{llll}
\hline Características físicoquímicas & Resultado \\
\hline${ }^{\circ}$ Brix & & 5 \\
Ácidez títulable & $(\%$ de ácido & \\
cítrico) & & & 1.8 \\
$\mathrm{pH}$ & & 4.2 \\
\hline
\end{tabular}

Fuente: Elaboración propia. 


\subsection{Caracterización de la pulpa de cocona}

Tabla 11. Características fisicoquímicas dela pulpa de cocona

\begin{tabular}{|l|l|}
\hline Características físicoquímicas & Resultado \\
\hline${ }^{\circ}$ Brix & 6.0 \\
\hline $\begin{array}{l}\text { Ácidez títulable (\% de ácido } \\
\text { cítrico) }\end{array}$ & 1.68 \\
\hline $\mathrm{pH}$ & 3.39 \\
\hline${ }^{\circ}$ Brix/ácidez & 3.57 \\
\hline
\end{tabular}

Fuente: Elaboración propia.

Tabla 12. Características fisicoquímicas del extracto de stevia

\begin{tabular}{ll}
\hline Características físicoquímicas & Resultado \\
\hline${ }^{\circ}$ Brix & 20 \\
$\mathrm{pH}$ & 5 \\
Densidad g/mL & 1.156 \\
\hline
\end{tabular}

Fuente: Elaboración propia.

\subsection{Caracterización del extracto de Stevia}

Tabla 13. Características sensoriales del extracto líquido de stevia.

\begin{tabular}{ll}
\hline Características sensoriales & descripción \\
\hline color & blanco \\
olor & Herbal \\
sabor & dulce \\
\hline
\end{tabular}

Fuente: Elaboración propia.

2.4 Caracterización del néctar de cocona endulzado con extracto de stevia y sacarosa.

En la tabla 14 se muestra un resumen de las pruebas de Duncan para los tres parámetros y en la tabla 15 se muestra un cuadro resumen del ANVA de los tres parámetros evaluados.
Tabla 14. resumen de las pruebas de Duncan para los tres parámetros

Duncan
\begin{tabular}{|l|l|l|l|}
\hline Tratamiento & Acidez & pH & Brix \\
\hline T7 & $1.8 \mathrm{a}$ & $4.15 \mathrm{a}$ & $5 \quad \mathrm{e}$ \\
\hline T6 & $0.5 \mathrm{~b}$ & $3.42 \mathrm{~b} \mathrm{c} \mathrm{d}$ & $18 \mathrm{a}$ \\
\hline T3 & $0.49 \mathrm{~b} \mathrm{c}$ & $3.43 \mathrm{~b} \mathrm{c}$ & $\begin{array}{l}6.65 \\
\text { d }\end{array}$ \\
\hline T2 & $0.47 \mathrm{~b} \mathrm{c}$ & $3.44 \mathrm{~b}$ & $\begin{array}{l}6.57 \\
\mathrm{~d}\end{array}$ \\
\hline T5 & $0.45 \mathrm{c}$ & $3.41 \mathrm{c} \mathrm{d}$ & $16 \mathrm{~b}$ \\
\hline T1 & $0.45 \mathrm{c}$ & $3.42 \mathrm{~b} \mathrm{c} \mathrm{d}$ & $\begin{array}{l}6.46 \\
\mathrm{~d}\end{array}$ \\
\hline T4 & $\begin{array}{l}0.4 \\
\mathrm{~d}\end{array}$ & $3.4 \mathrm{~d}$ & $14 \quad \mathrm{c}$ \\
\hline
\end{tabular}

Tabla 15. resumen del ANVA de los tres parámetros evaluados

\begin{tabular}{lcllll} 
& \multicolumn{5}{c}{ F.C } \\
F.V & G.L & $\begin{array}{l}\text { \% de } \\
\text { Acidez }\end{array}$ & pH & Brix & F.T \\
Tratamiento & 6 & $789.64 *$ & $1602.2 *$ & $244.66 *$ & 2.85 \\
Error & 14 & & & & \\
Total & 20 & & & & \\
ANVA & & 2.0182991 & 0.33914067 & 5.767853 \\
& & & & & \\
& C.V & 5.76785341 & &
\end{tabular}

De acuerdo a los resultados mostrados en las tablas 14 y 15 , existe diferencia significativa $(\alpha=0.05)$ entre los tratamientos analizados; es decir, al menos uno de los tratamientos difiere significativamente de los otros. Por lo tanto, se continuó con la prueba de Duncan para determinar cuáles son los tratamientos que difieren significativamente.

De acuerdo a los resultados que se observan en la tabla 14 y 15 . El tratamiento con mayor P.H es el tratamiento $\mathrm{S} 7$ con $0.0 \%$ extracto de cocona porque la fruta cuando está madura ya tiene contenido adecuado de azúcar.

De acuerdo a los resultados mostrados en las tablas 14 y 15 , se puede decir que el mejor tratamiento en brix es el tratamiento S6. $(0.7 \%$ de extracto de sacarosa) indicando que este tratamiento es más dulce y menos ácido. De acuerdo a los resultados de las tablas 14 y 15 , que el mejor tratamiento es el S4 ( $0.3 \%$ de extracto de sacarosa) que es el tratamiento con menor acidezy por lo tanto tiene el mejor sabor. 


\section{CONCLUSIONES}

Se concluye que no existe efecto significativo de la concentración de extracto de stevia sobre las características sensoriales (color, olor y sabor) en néctar de cocona; a partir de la prueba no paramétrica de Friedman a un nivel de significancia de 5\% realizada a los resultados obtenidos del análisis sensorial de los atributos color, olor y sabor de siete tratamientos de néctar de cocona endulzados con extracto de stevia. Así mismo, a partir de la prueba duncan a un nivel de significancia $\alpha=0.05$, se concluye que los tratamientos con $0.3 \%, 05 \%$ y $0.7 \%$ de extracto de stevia y extracto de sacarosa; difieren significativamente en sus valores de ph y acidez. Los siete tratamientos diferentes significativamente en cuanto a los grados ${ }^{\circ}$ Brix. Se puede decir que el mejor tratamiento de los siete evaluados en la presente investigación es el tratamiento $\mathrm{S}_{3}$ con $0.7 \%$ de extracto de stevia y $\mathrm{S}_{6}$, con extracto de sacarosa.

\section{AGRADECIMIENTOS}

A los encargados del laboratorio de la Escuela Académico Profesional de Industrias Alimentarias de la Universidad Nacional de Jaén por facilitarme los laboratorios y los medios necesarios para la ejecución dela presente investigación.

\section{REFERENCIAS BIBLIOGRÁFICAS}

Anzaldúa, E y Morales, A. (1994). La evaluación sensorial de los alimentos en la teoría y la práctica. Editorial Acribia. Zaragoza, España. Pp. 85-90.

Barrera, J. Páez. D. Oviedo. E. (1994). Conservación de pulpa de cocona (Solanum sessiliflorum Dunal) por diferentes métodos. Tratamientos térmicos y concentración. Instituto Amazónico de investigaciones científicas SINCHI. Florencia, Caquetá, Colombia. Pp 250.
Andrade, J.S.; Rocha, I.M.A.; Silva Filho, D.F. (1977). Características físicas y composición. Pp. 115.

Bello Gutiérrez, j. (2000). Ciencia bromatológica: principios generales de los alimentos. Madrid, España. Ediciones días de Santos, s. a. 581 p.

Castillo, M y Rojas, P. (2005). Determinación de las Propiedades en Zumo y Néctar Empleados en un Programa en Visual Basic. Chimbote-Perú. Pp 48.

Camacho. G. (1998). Deshidratación osmótica de frutas. En: memorias del curso sobre deshidratación de frutas y hortalizas. Universidad Nacional de ColombiaICTA. Bogotá. Pp 115.

Instituto Colombiano de Ciencia y Tecnología de alimentos, (1986). Elaboración de mermeladas. En: Memorias del curso Obtención y conservación de pulpas de frutas. Instituto Colombiano de Ciencia y Tecnología de alimentos-ICTA. Bogotá. Pp 325.

Cortés, 1 (2012) Análisis de crecimiento del cultivo de estevia (Stevia rebaudiana) con proyección agroindustrial en el valle del Cáuca. Tesis de grado. Ingeniería agroindustrial. Facultad de Ingeniería. Universidad de San Buenaventura Cali. Pp 150.

Erazo, Y. (1996). Lulo Amazónico. (Solanum sp) Corpoica Reg. 10. Florencia. Caquetá.

Fajardo, M. Murcia, S. (1998). Determinación del momento óptimo de cosecha y e laboración de productos osmodeshidratados de la cocona (Solanum sessiliflorum Dunal) en el piedemonte Caqueteño. Tesis Facultad de Ingeniería de Alimentos. Universidad Jorge Tadeo Lozano. Bogotá. 
Centro de investigación, educación y desarrollo. Procesamiento de alimentos para pequeñas y micro empresas agroindustriales. [En línea].Jaén, octubre de 2015. Disponible en: http://www.ciedperu.org/cendoc/manua les/nectar.pdf.

Costell, E. (2005). El Análisis Sensorial en el Control y Aseguramiento de la Calidad de los Alimentos. Centro Tecnológico Nacional de la Conserva y la Alimentación. Valencia, España. Pp 320.

European Food Safety Authority (EFSA). (2010). Scientific Opinion on the safety of steviol glycosides for the proposed uses as a food additive. EFSA Journal 8: 15-37.

Edac, Manual técnico de producción de stevia de la Equipo de desarrollo agropecuario de Cajamarca (EDAC). Disponible en www.incagro.gob.pe/a p c - a a files/.../Manual_T_cnico_de_Stevia.pdf .(Accesado 17/10/2015).

FAO. (2010). Steviol Glycosides. "Combined Compendium of Food Additive Specifications" Disponible en http://www.fao.org/ag/agn/jecfaadditives/details.html?id=898. (Accesado 17/01/2016).

González, A. (2011). Aproximación a la comprensión de un endulzante natural alternativo, la Stevia rebaudiana Bertoni: producción, consumo y demanda potencial. Agroalimentaria 17: Pp. 57-69.

Gonzales-Moralejo, A. (2011). Aproximación a la comprensión de un endulzante natural alternativo, la Stevia rebaudiana Bertoni: producción, consumo y demanda potencial. Agroalimentaria 17: Pp. 57-69.

Indecopi (1995). Norma Técnica Peruana “Néctares de Frutas”N²02.083.Lima Perú.
Indecopi (2003). Norma Técnica Peruana "Generalidades de jugos y Néctares" $\mathrm{N}^{\mathrm{O}}$ 203-011.Lima-Perú.

Icontec. (1990). Normas Técnicas para la elaboración de Mermeladas, Néctares y Yogur. Instituto Colombiano de Normas Técnicas. Bogotá.

Instituto Amazónico de Investigaciones Científicas Sinchi. Universidad de la Amazonia. Pronatta. (2001). Bases técnicas para el desarrollo de la agroindustria de frutas nativas en la Amazonia Occidental Colombiana.

Kujur, R.; Singh, V.; Ram, M.; Yadava, H.; Singh, K.; Kumari, S.; Roy, B. (2010). Antidiabetic activity and phytochemical screening of crude extract of Stevia rebaudiana in alloxan-induced diabetic rats. Pharmacognosy Res 2: 258-263.

Lemus-Mondaca, R.; Vega-Gálvez, A.; ZuraBravo, L.; Ah-Hen K. (2012). Stevia rebaudiana Bertoni, source of a highpotency natural sweetener: A comprehensive review on the biochemical, nutritional and functional aspects. Food Chemistry 132: 1121-1132.

Núñez, E. (2011). Stevia rebaudiana Bertoni, un sustituto del azúcar. Área Ciencia de las Plantas y Recursos Naturales Maestría en Producción Vegetal - Ciclo de Seminarios. Pp 97.

Postharvest Biology and Technology of Tropical and Subtropical Fruits Cocona to Mango.A volume in Woodhead Publishing Series in Food Science, Technology and Nutrition. 2011, Pages $1-7,80$.

Panes, M y Rua, D. (2009). Proyecto de Néctar de Maracuyá Universidad Nacional Mayor de San Marcos - Lima-Perú. :Pp .150. 
Paytan, S.F. (1997). Cultivo de frutos nativos amazónicos: Manual para el extensionista. Lima: Tratado de Cooperación Amazónica, p. 71-76.

Región de Loreto. Cocona. [En línea]. Jaén, octubre de 2015. Disponible en: http://www.regionloreto.gob.pe/amazon ia/libros/51/5100001.htm\#I1.Campos Saravia, Karina.

Ribeiro, B.G. (1990). Clasificación de los suelos y horticultura Desana. In: Ethnobiology: Implications and applications. Proceedings of the first International Congress of Ethnobiology/ Darrell Adison Posey, William Leslie Overal, Charles Roland Clement, Mark J. Plotkin, Elaine Elisabetsky, Clarice Novaes de Barros: - Belém, Museu Emílio Goeldi, 1990.p. 11-26.

Renwick, A.; Tarka, S. (2008). Microbial hydrolysis of steviol glycosides. Food and Chemical Toxicology 46: 70-74.

Salick, J. (1988). Cocona (Solanum sessiliflorum) Production, Nutrition and Breeding, potentials of the peach tomato, an underexploited crop of the Peruvian tropics. USAID. Perú.

Storti, E.F. (1988). Biología floral de Solanum sessiliflorum Dunal, var. sessiliflorum, en la región de Manaus. Acta Amazónica, 18:56-68.

Vargas Y, y Pisfil, E. (2008).Estudio químico bromatológico y elaboración de néctar de Mespilus germánica L. (níspero de palo) procedente de la provincia de Vilca Huamán, departamento de Ayacucho. Tesis Universidad Nacional mayor de San Marcos - Lima - Perú.

Villachica, J. Carvalho, E.U., Müller, C.H., Díaz, C. y Almanza M. (1996). Frutales y Hortalizas promisorias de la Amazonia. Lima, Perú, Tratado de Cooperación Amazónica -Secretaria Pro-Tempore. $367 p$.
Villachica, H. (1995). El cultivo del Camú Camú (Myrciaria dubia (H.B.K.) McVaugh) en el Amazonas Peruano. Lima, Perú: Secretaria Pro-Tempore, Tratado de Cooperación Amazónica. 95 p.

Yong-Heng, Y.; Su-zhen, H.; Yu-lin, H.; Hai-yan Y.; Chun-sun, G. (2014). Base substitution mutations in uridinediphosphate-dependent glycosyltransferase 76G1 gene of Stevia rebaudiana causes the low levels of rebaudioside A Mutations in UGT76G1 A key gene of steviol glycosides synthesis. Plant Physiol Biochem 80: 220-225.

\section{CORRESPONDENCIA}

Irma Rumela Aguirre Zaquinaula

irmarumela@hotmail.com

Universidad Nacional de Jaén 